\title{
SOLUTIONS AND DISCUSSIONS OF THIN FILM UNDERGOING THE NONLINEAR PEELING
}

Yueguang Wei, Siqi Shu and Ying Du

LNM, Institute of Mechanics, Chinese Academy of Sciences, Beijing 100080, P.R. China

\begin{abstract}
Based on the bending model, three double-parameter criteria characterizing thin film peeling process are introduced and analyzed in detail. Three double-parameter criteria include: (1) the interfacial fracture toughness and the separation strength, (2) the interfacial fracture toughness and the interfacial crack tip slope angle of thin film, and (3) the interfacial fracture toughness and the critical von Mises effective strain of thin film at crack tip. Based on the three double-parameter criteria, the thin film nonlinear peeling problems are solved analytically for each case. The results show that the solutions of thin film nonlinear peeling based on the bending model are very sensitive to the model parameter selections. Through analyses and comparisons for different solutions, a connection between solutions based on the bending models and based on the two-dimensional elastic-plastic finite element analysis is obtained.
\end{abstract}

\section{INTRODUCTION}

Most advanced materials are inseparable with thin films. Thin film, with its particular characteristic, has been widely applied to the surface and interfacial engineering areas. The material behaviors of the thin film systems mainly depend on the interfacial adhesion property (strength) between the thin film and substrate. In order to evaluate the adhesion behaviors, a simple test method, peel test, was designed in fifty years ago [1]. Due to the good advantages of the test method, such as simply operating, the test method has been widely applying to many research regions [2-4]. Specifically, when both the thin film and substrate are elastic materials, the interfacial adhesion toughness can be obtained through directly measuring peeling force in peel test. However, with development of the research and application, it has been noted that when thin film is a ductile material, the measured peeling force is often much larger than the interfacial adhesion toughness. The phenomenon is come from the plastic dissipation due to plastic loading and unloading deformation of the thin film. In order to model the peeling force (or energy release rate) increase due to plastic dissipation, Kim and his collaborators [5,6] presented a bending model to predict the plastic dissipation during the process of the thin film peeling. Within the following decade after bending model was presented, most analyses related to the ductile thin film peeling adopted the bending model of Kim et al, e.g., [7,8]. However, Wei and Hutchinson [9] adopted a different method from that of Kim et al in analyzing the elastic-plastic thin film peeling problems. In their analysis, the thin film delamination process was simulated by using the two-dimensional elastic-plastic finite element method (FEM), except the detached part of thin film, which was described still by bending model. They obtained a different result from that of bending model, qualitatively and quantitatively. In the present research, in order to explore the connection of bending model solution with elastic-plastic FEM solution, three double-parameter criteria based on the bending model will be adopted to describe the peeling process of thin film, and the solutions are carried out for each double-parameter 
criterion. By comparing the present results with that of FEM from Wei and Hutchinson model [9], a primary connection of both the bending model and the two-dimensional FEM model is obtained. Furthermore, the reliability of the bending model is assessed.

\section{BENDING MODELS AND DELAMINATING CRITERIA IN PEEL TEST}

Delaminating process of elastic-plastic thin film in peel test can be described by figure 1(a). The thin film undergoes the delamination and plastically loading and unloading process under the act of the peel force P. The cross-section of the thin film is from a free-stressing state to the loading and unloading processes, as described by OABCDEF, in sketch figure 1(a).

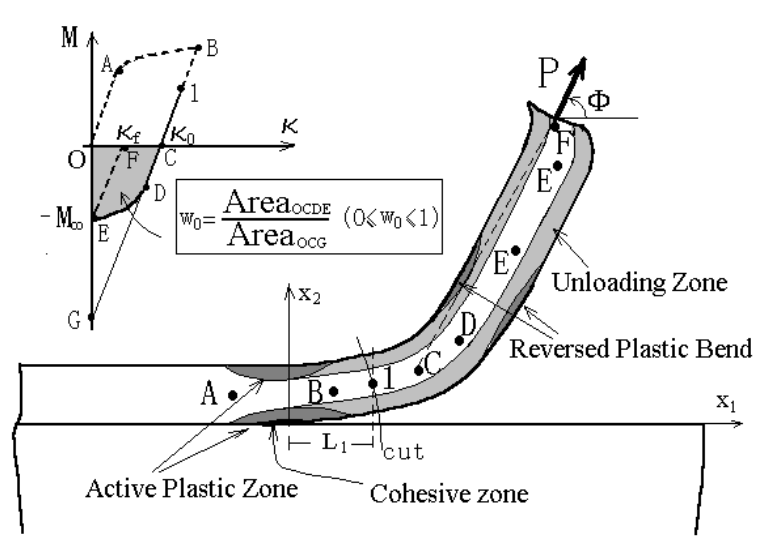

(a). Peel test

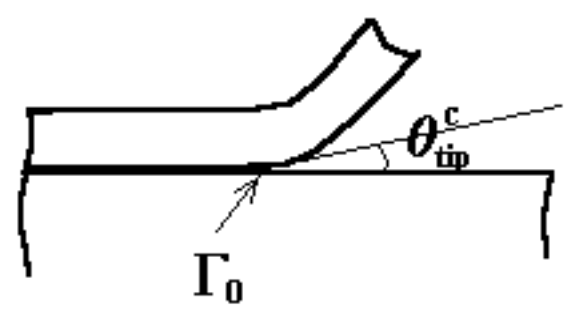

(c). $\left(\Gamma_{0}, \theta_{\text {tip }}^{c}\right)$ criterion

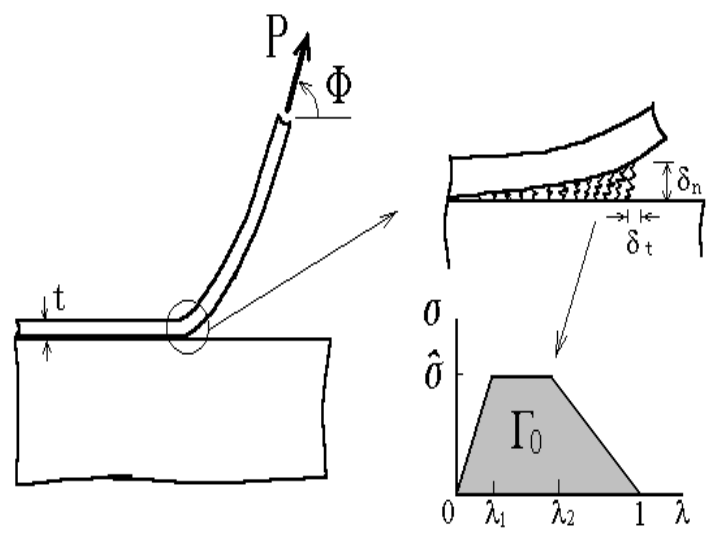

(b). $\left(\Gamma_{0}, \hat{\sigma}\right)$ criterion

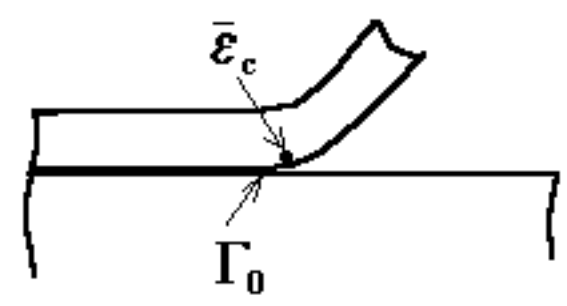

(d). $\left(\Gamma_{0}, \bar{\varepsilon}_{c}\right)$ criterion

Figure 1. Peel test sketch figure (a) and three simplified double-parameter models (b)-(d).

The process of the ductile thin film peeled and delaminated along substrate interface can be characterized by the double-parameter criterion (for elastic delamination case, single-parameter criterion is needed). Two independent parameters are needed to characterize the main characters here, the interfacial adhesion property and the plastic dissipation of the system. In the present research, three double-parameter criteria will be used respectively for describing the elastic-plastic peeling process, which are given in figure 1 (b), (c) and (d), respectively.

For the thin film peeling process, the relation among the peeling force $\mathrm{P}$ per unit width of 
thin film (or energy release rate of system), the interfacial adhesion toughness $\Gamma_{0}$, as well as the geometrical and physical parameters of thin film and substrate is usually concerned. Under steady-state delamination condition, the relation can be written as:

$$
P(1-\cos \Phi)=\Gamma_{0} \quad(\text { elastic peeling }) ; \quad P(1-\cos \Phi)=\Gamma_{0}+\Gamma^{P} \quad \text { (elastic-plastic peeling) }
$$

where $\Gamma^{P}$ is the plastic dissipation. Based on the stress-strain analysis for thin film, one can obtain the fundamental relations of the thin film undergoing the nonlinear bending, furthermore, one can also obtain the plastic dissipation relation for $\Gamma^{P}$, as given in next section.

\section{FUNDAMENTAL RELATIONS}

Kim and Aravas [5] derived out the fundamental relations based on the bending model for elastic-plastic thin film bending under the incompressible conditions $(v=1 / 2)$. The rigorous derivation based on the general case of the compressible elastic-plastic conditions is given by [10]. The relations of moment and curvature respectively for elastic, plastic and unloading cases can be dictated as follows:

$$
\frac{M}{M_{0}}=\frac{2 \kappa}{3 \kappa_{e}} ; \quad \frac{M}{M_{0}}=\left\{\frac{2}{3}-\frac{2}{N+2} \gamma\right\} \frac{1}{\left(\kappa / \kappa_{e}\right)^{2}}+\frac{2}{N+2} \gamma\left(\frac{\kappa}{\kappa_{e}}\right)^{N} ; \quad \frac{M}{M_{0}}=\frac{2}{3} \frac{\kappa-\kappa_{0}}{\kappa_{e}},
$$

and curvature relation: $\kappa=\sqrt{[1-\cos (\Phi-\theta)] \frac{2 P}{B}+\left(1-w_{0}\right) \kappa_{0}^{2}}, \theta_{B} \approx \theta_{t i p} \leq \theta \leq \theta_{C}$.

where $M_{0}=\frac{3}{2} M_{e}$ is the limit bending moment for elastic-perfectly plastic material; $M_{e}$ and $\kappa_{e}$ are the elastic limit moment and elastic limit curvature, respectively,

$$
M_{e}=\frac{2}{3} M_{0}=\frac{\sigma_{Y} t^{2}}{6 \sqrt{1-v+v^{2}}}, \quad \kappa_{e}==\frac{2\left(1-v^{2}\right) \sigma_{Y}}{E t \sqrt{1-v+v^{2}}}, \quad \gamma=2{\sqrt{\frac{1}{3}\left(1-v+v^{2}\right.}}^{1-N}(1-v)^{N}
$$

$B=E t^{3} / 12\left(1-v^{2}\right)$ is the bending modulus; $w_{0} \quad\left(0 \leq w_{0} \leq 1\right)$ is defined in figure 1(a) which characterizes the inversely plastic behavior (or Bauschinger effect); $\theta_{\text {tip }}$ is the crack tip slope angle at thin film delamination; $N$ is material strain hardening exponent. For incompressible material $v=0.5$ and $\gamma=1$, expression (2) comes to the result of Kim and Aravas [5].

Suppose that substrate is rigid or Young's modulus of substrate is much larger than that of thin film, by means of formulas (2) ( $M-\kappa$ relations in sketch of figure 1(a)), one can obtain the plastic dissipation relation through calculating the area within the circuit OABCDEO under $M-\kappa$ curve,

$$
\begin{aligned}
\Gamma^{P}=\frac{1}{2} M_{e} \kappa_{e}-\frac{1}{2} M_{B}\left(\kappa_{B}-\kappa_{0}\right)+\left(\frac{2}{3}-\frac{2}{N+2} \gamma\right) M_{0}\left(\kappa_{e}-\frac{\kappa_{e}^{2}}{\kappa_{B}}\right) \\
+\frac{2 \gamma}{(N+1)(N+2)} M_{0}\left(\frac{\kappa_{B}^{N+1}}{\kappa_{e}^{N}}-\kappa_{e}\right)+\frac{1}{2} B \kappa_{0}^{2} w_{0}
\end{aligned}
$$

\section{BENDING MODEL SOLUTIONS OF THIN FILM NONLINEAR PEELING}




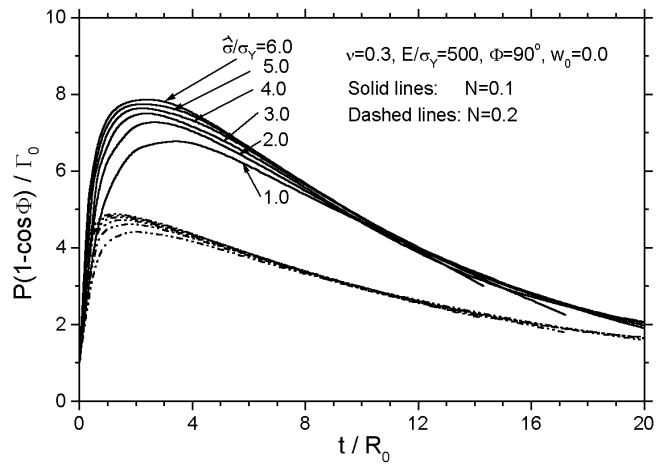

(a)

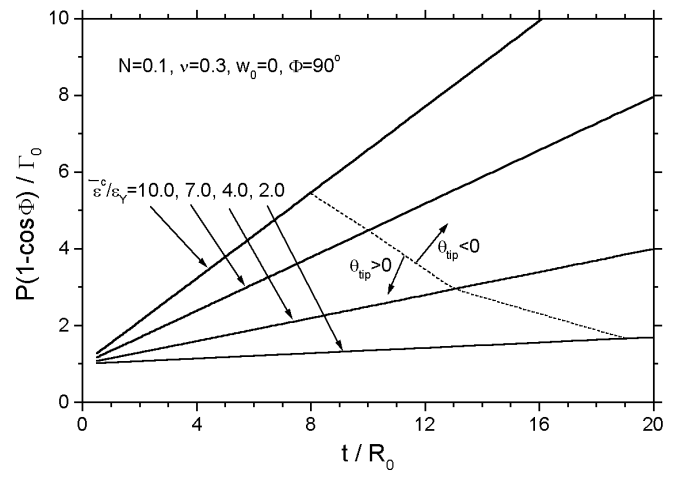

(c)

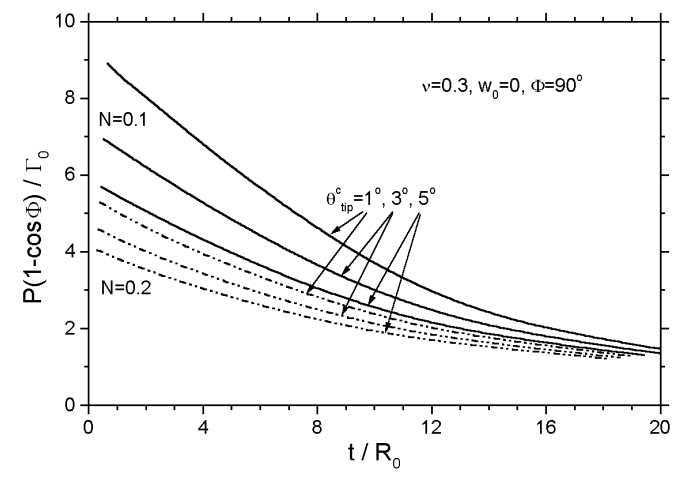

(b)

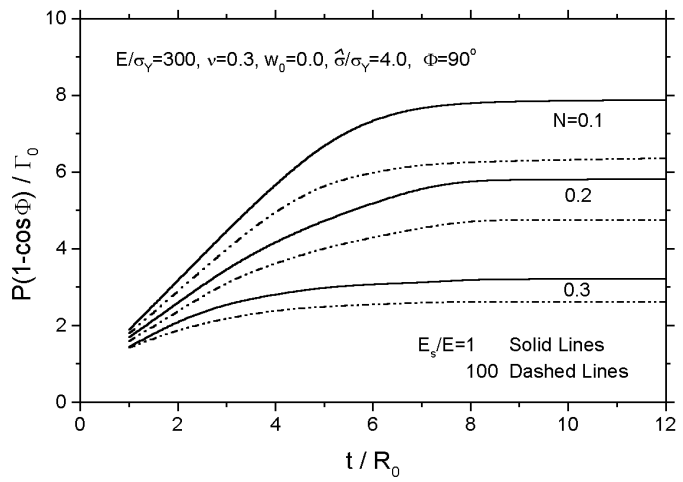

(d)

Figure 2. Results based on three double-parameter criteria ((a)-(c)) and two-dimensional finite element method $((d))$.

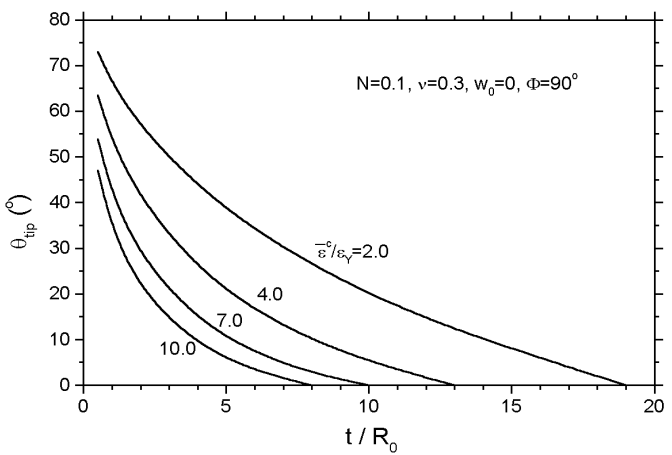

(a)

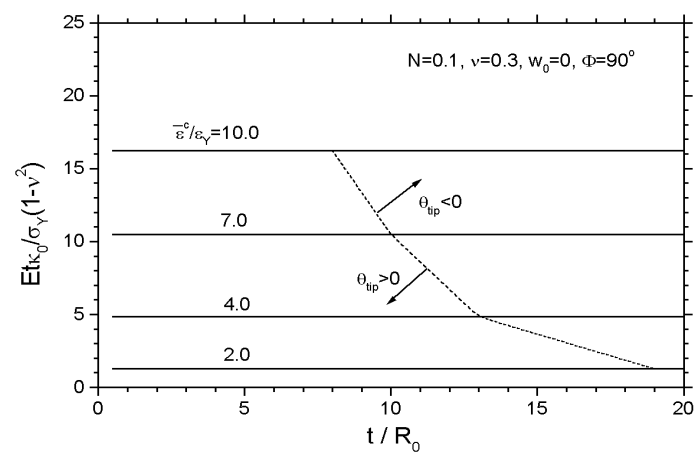

(b)

Figure 3. Variations of the thin film inclined angle at tip and the residual curvature with thin film thickness based on the criterion $\left(\Gamma_{0}, \bar{\varepsilon}_{c}\right)$ 
Based on three double-parameter criteria, the peel force can be solved for peeling process. The solving procedures can be dictated as follows. Firstly, from (3) a relation among the parameters $\left(\kappa_{B}, P, \theta_{\text {tip }}=\theta_{B}, \kappa_{0}\right)$ can be obtained to each criterion. Then the parameters $\left(M_{B}, \kappa_{B}, \kappa_{0}\right)$ can be solved simultaneously with equations (2) for $M=M_{B}$ and $\kappa=\kappa_{B}$. Finally, the peel force variations with related parameters can be attained from (1) and (5).

The solution forms by adopting three double-parameter criteria can be given through independent parameters by dimensional analysis. They are dictated as

$$
\begin{aligned}
& \left\{\begin{aligned}
\frac{P(1-\cos \Phi)}{\Gamma_{0}} & =f_{1}\left(E / \sigma_{Y}, \hat{\sigma} / \sigma_{Y}, N, v, t / R_{0}, w_{0}, \Phi\right) \\
\theta_{t i p} & =r_{1}\left(E / \sigma_{Y}, \hat{\sigma} / \sigma_{Y}, N, v, t / R_{0}, w_{0}, \Phi\right)
\end{aligned} \quad \text { (for }\left(\Gamma_{0}, \hat{\sigma}\right)\right. \text { criterion); } \\
& \left.\frac{P(1-\cos \Phi)}{\Gamma_{0}}=f_{2}\left(N, v, t / R_{0}, w_{0}, \Phi, \theta_{t i p}^{c}\right) \quad \text { (for }\left(\Gamma_{0}, \theta_{t i p}^{c}\right) \text { criterion }\right) \\
& \left\{\begin{aligned}
\frac{P(1-\cos \Phi)}{\Gamma_{0}} & =f_{3}\left(N, v, t / R_{0}, w_{0}, \Phi, \bar{\varepsilon}_{c} / \varepsilon_{Y}\right) \\
\theta_{t i p} & =r_{3}\left(N, v, t / R_{0}, w_{0}, \Phi, \bar{\varepsilon}_{c} / \varepsilon_{Y}\right)
\end{aligned}\right.
\end{aligned}
$$

where a length parameter $R_{0}$ is introduced and is defined as $R_{0}=E \Gamma_{0} / 3 \pi\left(1-v^{2}\right) \sigma_{Y}^{2}$, which characterizes the plastic zone size at crack tip under the small scale yielding case. $\theta_{\text {tip }}$ is crack tip slope angle. $\varepsilon_{Y}=\sigma_{Y} / E$ is yielding strain.

The solutions based on three double-parameter criteria $\left(\Gamma_{0}, \hat{\sigma}\right),\left(\Gamma_{0}, \theta_{\text {tip }}^{c}\right)$ and $\left(\Gamma_{0}, \bar{\varepsilon}_{c}\right)$ are given in figure 2(a)-(c), respectively. Results in figure 2 show the curves of the normalized energy release rate (or peeling force) vs. normalized thin film thickness under elastic-plastic steady-state delamination. If thin film deformation is elastic, $P(1-\cos \Phi) / \Gamma_{0}=1$, see first relation of formula (1). Clearly, the energy release rate is remarkably enlarged by the plastic dissipation. From figure 2(a), the variation of the normalized peeling force (energy release rate) $P(1-\cos \Phi) / \Gamma_{0}$ with thin film thickness can be described as follows. Its value tends to 1 when thin film thickness is very small, i.e., the effect of plastic dissipation can be neglected. As thin film thickness increases, energy release rate increases sharply and obtains a maximum value at about $t / R_{0}=2$. The plastic dissipation decreases gradually as thin film thickness increases further. The results shown in figure $2(\mathrm{~b})$ are based on the $\left(\Gamma_{0}, \theta_{\text {tip }}^{c}\right)$ criterion. From the results, the variation of $P(1-\cos \Phi) / \Gamma_{0}$ vs. $t / R_{0}$ is much different from that based on the double-parameter criterion $\left(\Gamma_{0}, \hat{\sigma}\right)$. Here, the value of $P(1-\cos \Phi) / \Gamma_{0}$ increases always as thin film thickness decreases. This trend is consistent with that given by Kim and his collaborators for incompressible material [5,6]. The results based on the $\left(\Gamma_{0}, \bar{\varepsilon}_{c}\right)$ criterion are given in figure 2(c). Obviously, the variation of $P(1-\cos \Phi) / \Gamma_{0}$ vs. $t / R_{0}$ is different from those shown above based on the other double-parameter criteria. In figure $2(\mathrm{c}), P(1-\cos \Phi) / \Gamma_{0}$ increases linearly with increasing $t / R_{0}$. The inclined angles of the straight line increase with increasing the critical von Mises effective strain at tip. In order to investigate the third criterion, figure 3(a) and (b) show the variations of the crack tip slope angle and the residual curvature. When thin film thickness is small, the slope angle is large. With increasing thin film thickness, the slope angle 
decreases sharply and transits to negative value from positive value. Obviously, the negative slope angle of thin film at tip violates the physical requirement. It implies that the criterion is failure and the predicted results, top part of the dashed line in figure 2(c), should be cut off. The reason can be interpreted in referring to figure 3(b) that when thin film thickness is small, the large critical Mises effective strain at crack tip is realized by large bending deformation (curvature) in thin film, however when thin film thickness is large, it is difficult or even impossible to realize the large bending curvature of thin film to meet with the high critical Mises effective strain, and in this case the criterion $\left(\Gamma_{0}, \bar{\varepsilon}_{c}\right)$ is failure.

\section{DISCUSSIONS TO BENDING MODEL SOLUTIONS}

The bending model solutions of thin film nonlinear peeling based on the three double-parameter criteria have been obtained in last section. Obviously, the bending model solutions are very sensitive to the selection of the governing parameters. This leads to a question: what is a reasonable selection of the governing parameter group for predicting the thin film nonlinear peeling by using the bending model? From the solutions based on the first and second double-parameter criteria, when normalized thin film thickness is smaller than about 5, $t / R_{0}<5$, the contribution of bending plastic dissipation to energy release rate is very large. This can be confirmed from variation of the residual curvature in reference [10]. However, from the solutions based on the criterion of the interfacial fracture toughness and the critical Mises effective strain, when thin film thickness is smaller than about $5, t / R_{0}<5$, the contribution of the bending plastic dissipation to energy release rate is obviously smaller than that based on the first or second criterion. Comparing the present results based on the bending model to the two-dimensional elastic-plastic finite element results [9] (shown in figure 2(d)), the bending model results based on the third double-parameter criterion have the similar variation trends and quantity to those of the elastic-plastic finite element calculation within the region of about $t / R_{0}<5$ (in figure 2(d), $E_{s}$ is Young's modulus of elastic substrate). However, as thin film thickness increases continuously, the finite element solution tends to the small scale yielding solution (insensitive to thin film thickness), while the bending model solution based on the double-parameter criterion is failure. Therefore, when thin film thickness is large, i.e., $t / R_{0}>5$, how to set up a governing parameter criterion based on the bending model will be a tough task to need to be explored in the future.

\section{REFERENCES}

1. Spies GJ. J. Aircraft Eng., 25, 64(1953).

2. Feliu-Baez R, Lockhart HE, Burgess G. Packaging Tech. Sci., 14, 63(2001).

3. Choi JW, Oh TS. J. Adhe. Sci. Tech., 15, 139(2001).

4. Asai H, Iwase N, Suga T. IEEE Trans. on Adv. Packaging, 24, 104(2001).

5. Kim KS, Aravas N. Int. J. Solids Struct., 24, 417(1988).

6. Kim J, Kim KS, Kim YH. J. Adhe. Sci. Tech., 3, 175(1989).

7. Kinloch AJ, Lau CC, Williams JG. Int. J. Fracture, 66, 45(1994).

8. Moidu AK, Sinclair AN, Spelt JK. J. Testing Evalu., 26, 247(1998).

9. Wei Y, Hutchinson JW. Int. J. Fracture, 93, 315(1998).

10.Wei Y, Hutchinson JW. In Encyclopedia of Comprehensive Structural Integrity, 8, eds. Gerberich WW and Yang W, Chapter 5, Elsevier Science Publisher, 181(2003). 